\title{
Biomimetics: Learning From Nature To Make Better Sensors
}

\author{
P.J. French, D.H.B. Wicaksono+ \\ EI-EWI-DIMES, TUDelft, Mekelweg 4, 2628CD Delft, The Netherlands \\ $+3 \mathrm{Me}$, TU Delft, Mekelweg 2, 2628CD, Delft, The Netherlands
}

\begin{abstract}
Nature has been the inspiration in art for centuries. In the $19^{\text {th }}$ Century there were a number of attempts to copy nature and apply the ideas to engineering. Unfortunately, this was often done without understanding the details of operation of the natural systems. In some cases engineering successes were based on natural systems without the inventor realising. In more recent years there has been a more detailed investigation of natural systems with the aim to learn from them and build improved sensors. Once again it is important to learn about the mechanisms and not try to copy directly. This paper will discuss a number of natural systems and how they can be implemented in both sensors and actuators.
\end{abstract}

\section{Introduction}

Nature can teach us many things in terms of sensors, actuators and systems. From the largest mammals to the smallest insects we can find complete sensor and actuator systems, all optimised for the application and perfectly adjusted for the scaling up or down. The art of biomimetics is to learn from nature and not try to copy directly, without understanding the function. Examples of this problem can be seen in the first attempts to fly. Early attempts tried to copy the global structure of birds, without understanding the aerodynamics. These were often based on a direct copy of the natural system on a macroscale (Figure 1).

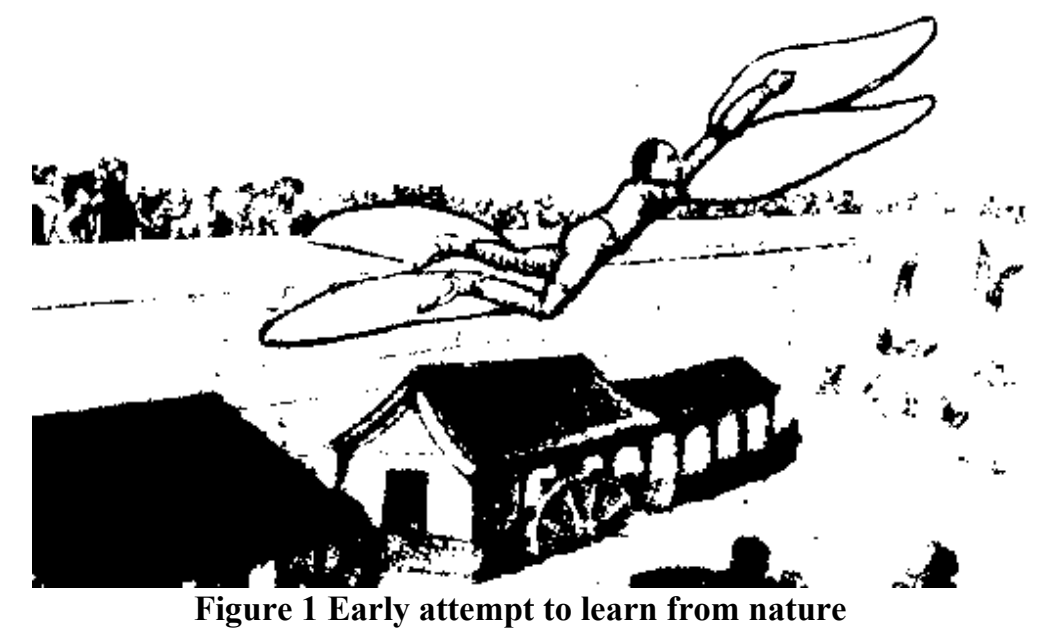


Since this time more has been learnt about the way in which birds fly and these have been applied to modern aviation. An example of this is the addition of extra tails on the end of the wings. This gives better stability and reduces vortices. This is shown in Figure 2.
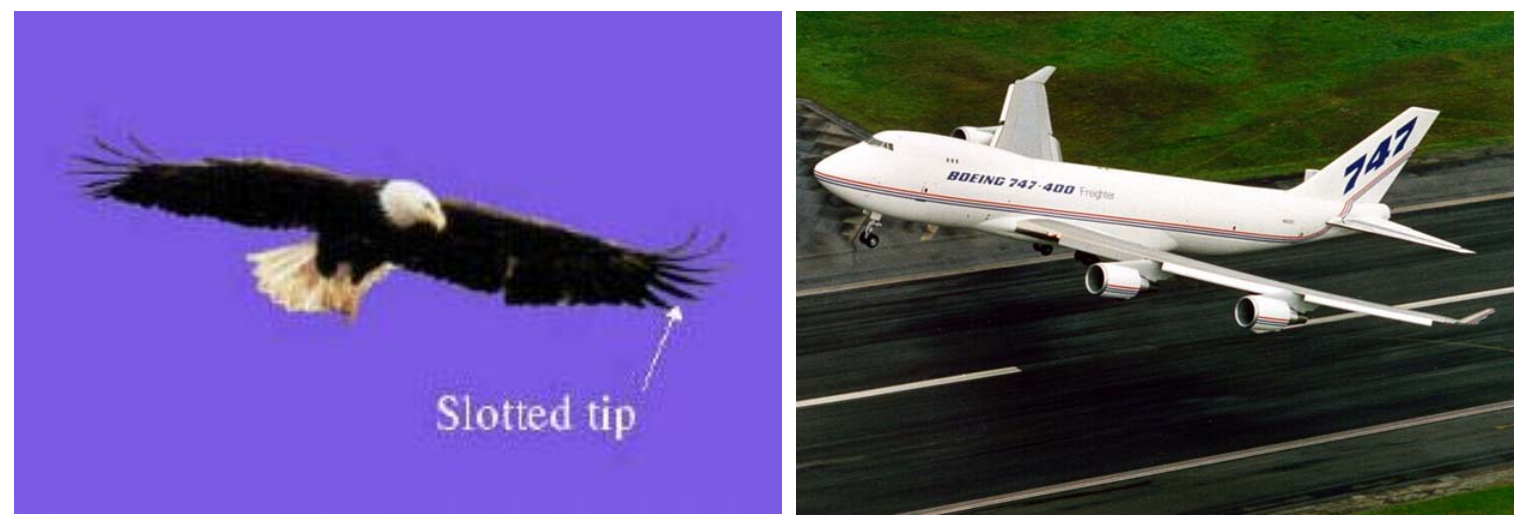

Figure 2 Example of biomimetics, aircraft wings

We also see examples in sport. Sharks are able to swim at high speed with high efficiency. One of the important factors is the shark's skin, where the roughness traps small air bubbles enabling the shark so swim more efficiently. This idea has been applied to swimming costumes with great success, with a number of world records being broken since their introduction. The structure of this is shown in Figure 3.

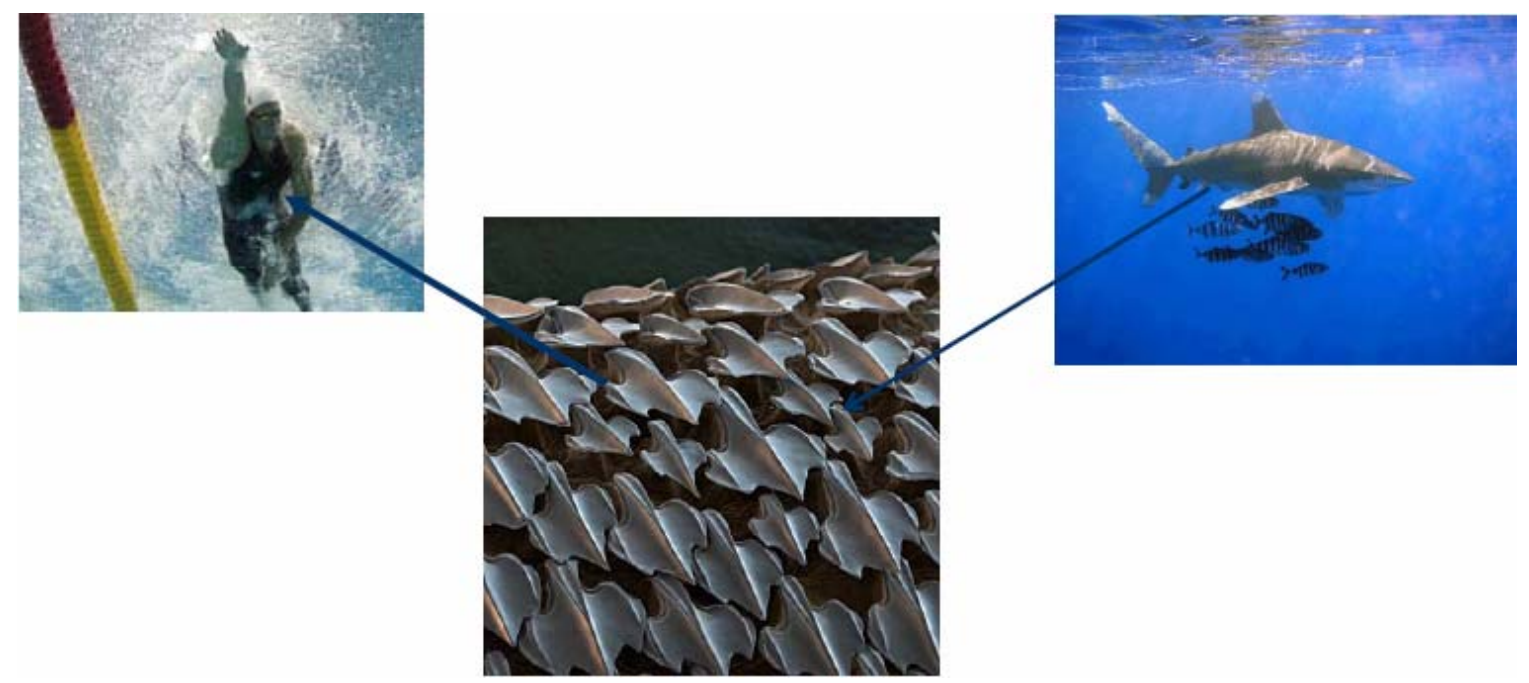

Figure 3 The use of a shark-skin like structure for swim suits.

Another example we find in everyday life. Velcro is a simple system, which allows to surfaces to stick to each other easily, for example for fastening shoes. This structure can be found in a number of plants to enable their seeds to stick to passing animals and be carried to another location. This basic structure can be seen in Figure 4.

In the field of sensors many examples can be found of structures, which can also be found in nature. In some cases the researchers were not aware of the natural devices, but increasingly research groups are looking to nature for their inspiration. Sharks use a type of stochastic process to be able to measure blood in very low concentrations. Excellent examples of compact sensor systems can be found in insects. They have compact gyroscopes for balance, crosswind sensors for stability in wind, highly accurate strain sensors and many more. This paper will look at a number of natural sensors, which 
can be used as inspiration for new sensors, although we have to keep in account that we are often using other materials and therefore the structure often cannot be copied directly.

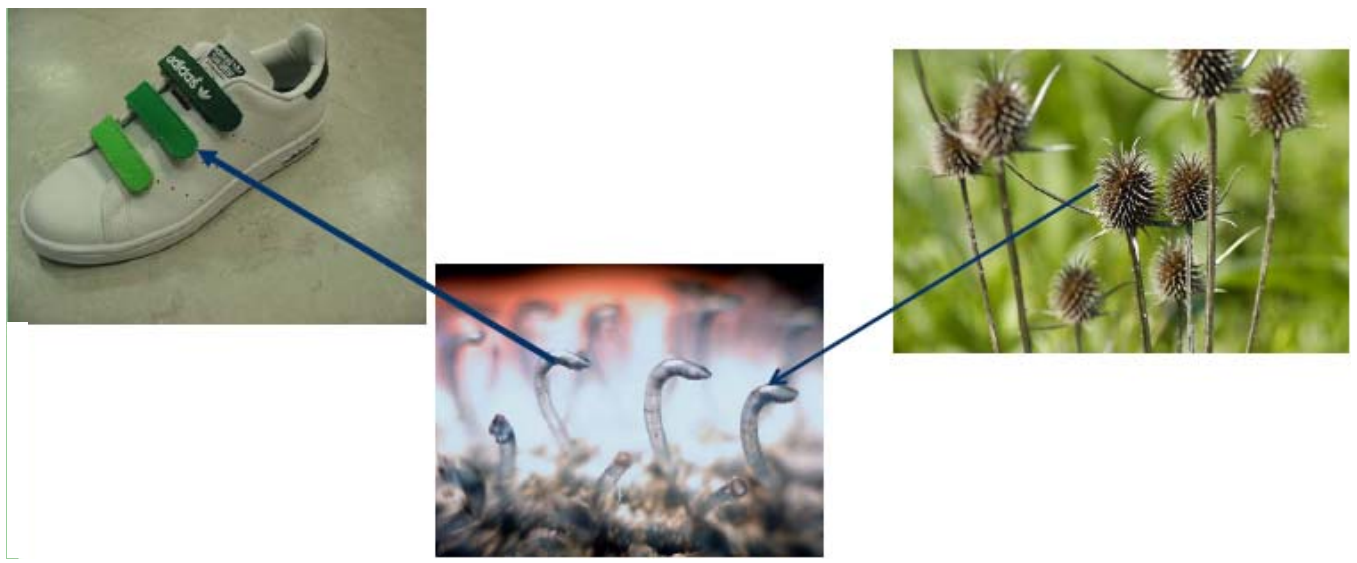

Figure 4 Velcro and its natural inspiration.

\section{Biomimetic sensors}

Many examples can be found where basic ideas found in nature can be applied in materials such as silicon. Many of these can be found in insects. Insects have made best use of scaling in fully integrated sensor and actuator systems. This section discusses a few examples, which have taken these systems.

\section{$\underline{\text { Strain sensor }}$}

Nature has long used highly sensitive strain sensors. Examples can be found in the insect world. Insects use a very small $(\sim 10-20 \mu \mathrm{m}$ in lateral dimension) special strain sensor called the campaniform sensillum. Campaniform sensillum is a sense organ in insects for detecting deformations or strains in its cuticle, or exoskeletal material [1]. The basic structure is shown in Figure 5. Its unique structure with a dome-shaped membrane in a hole, yields strain amplification that enables more sensitive strain detection, even with a high rigidity, i.e. a Young's Modulus of $\sim 2 \mathrm{GPa}$ of a membrane with diameter $\sim 20$ $\mu \mathrm{m}$, and thickness of $\sim 2 \mu \mathrm{m}$. A cross-section of the structure is given in Figure 6 . When developing a sensor based on this, the first task is to understand the structure and the function of each component. The second stage is to develop a sensor, which makes use of these features, but uses other materials.

The first view of the silicon version, shown in Figure 7, looks rather different. However, this sensor makes use of the sunken membrane. The read-out system is quite different, since in this version the strain in measured using piezoresistors on the membrane. This basic structure has shown that the strain is amplified, and thus the advantages of the biological sensor have been transferred to the silicon sensor world. An improved version is shown in Figure 8, in which the dome structure of the device is used. 

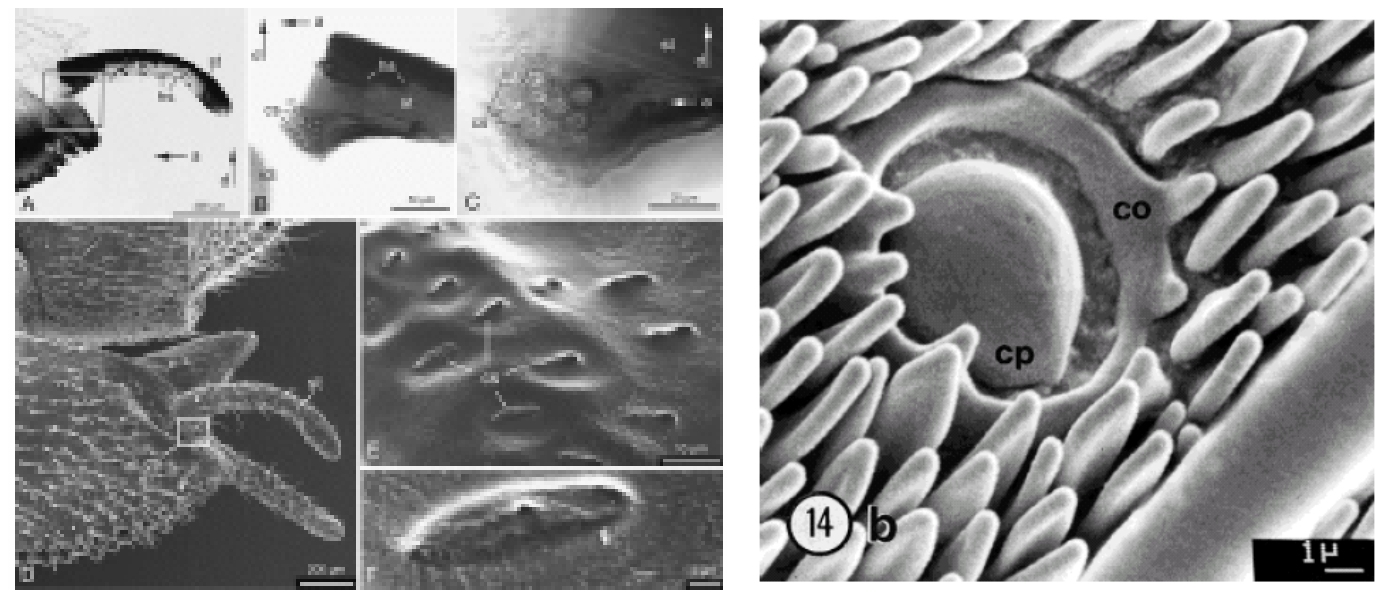

Figure 5 (left) Scanning electron microscopy from females of damselflies Lestes sponsa. The campaniform sensilla on the lateral side of the articulation between the stylus and lateral ovipositor valvula. cs, campaniform sensilla; st, stylus. From [2]. Copyright (2002 Elsevier Science Ltd.), reprinted with permission of Elsevier Science Ltd.(right) SEM image of campaniform sensillum on an antennal branch of the silkmoth, Antheraea polyphemus. From [3]. Copyright (1997 WileyLiss, Inc.), reprinted with permission of Wiley-Liss, Inc., a subsidiary of John Wiley \& Sons, Inc.

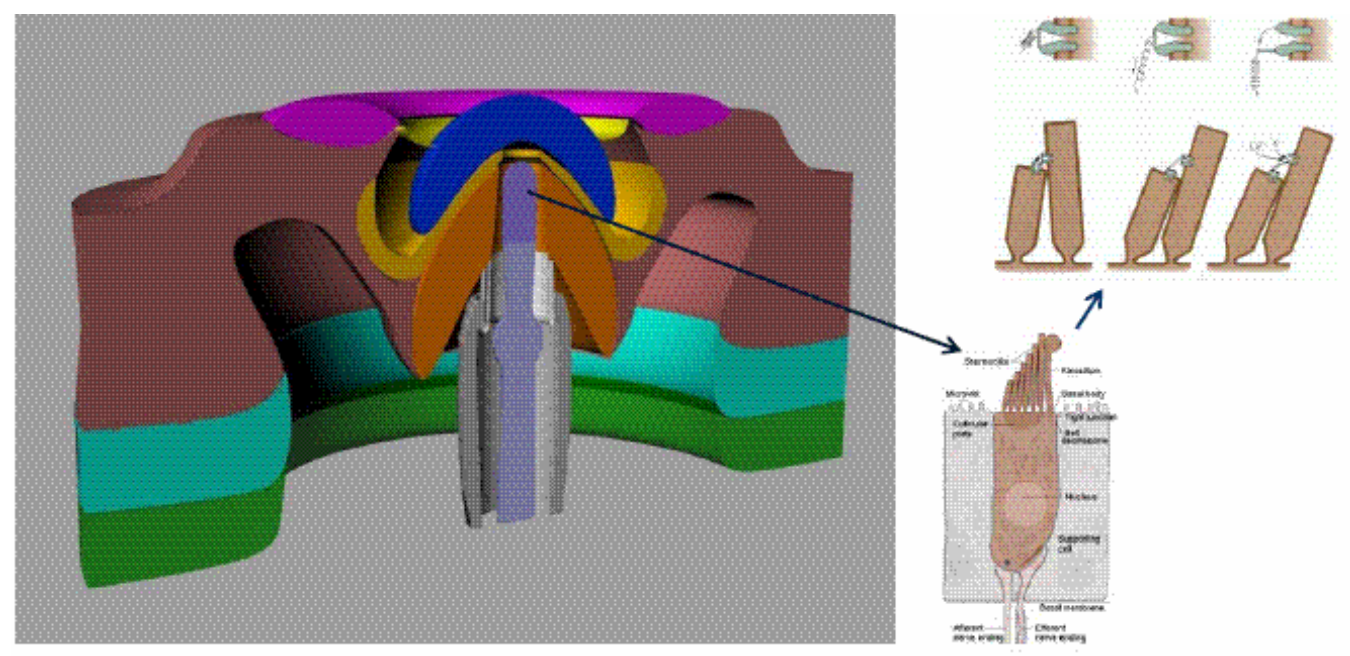

Figure 6 (left) 3-D cross section illustration of Campaniform sensillum found in Blowfly (Calliphora vicina) (adapted from [4]), (right) details of the sensing element.

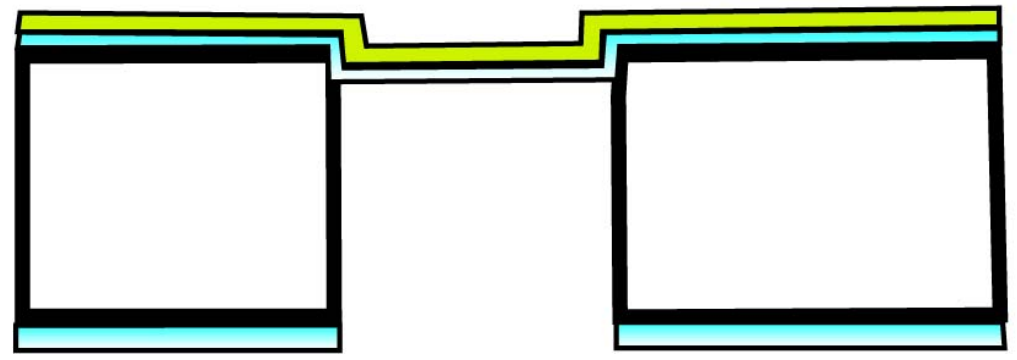

Figure 7 Simple version of the strain sensor utilising the sunken membrane. 
Figure 8 Cross section of a silicon version of the strain sensor.

\section{$\underline{\text { Flow sensors }}$}

Nature shows us many examples of flow sensors both for gasses and fluids. A hair is a good example of a flow sensor design for air-flow. This can be for hearing, detecting cross-wind, detecting predators. For detecting predators, crickets have sensitive mechanoreceptive sensory hairs, which are sensitive to low-frequency sound. These hairs are situated on the back of the cricket's body. Using this the cricket can pinpoint lowfrequency sound from any given direction, by using the combined neural information of all sensory hairs [6]. The natural system and a mechanical model with which we can develop devices are given in Figure 9.
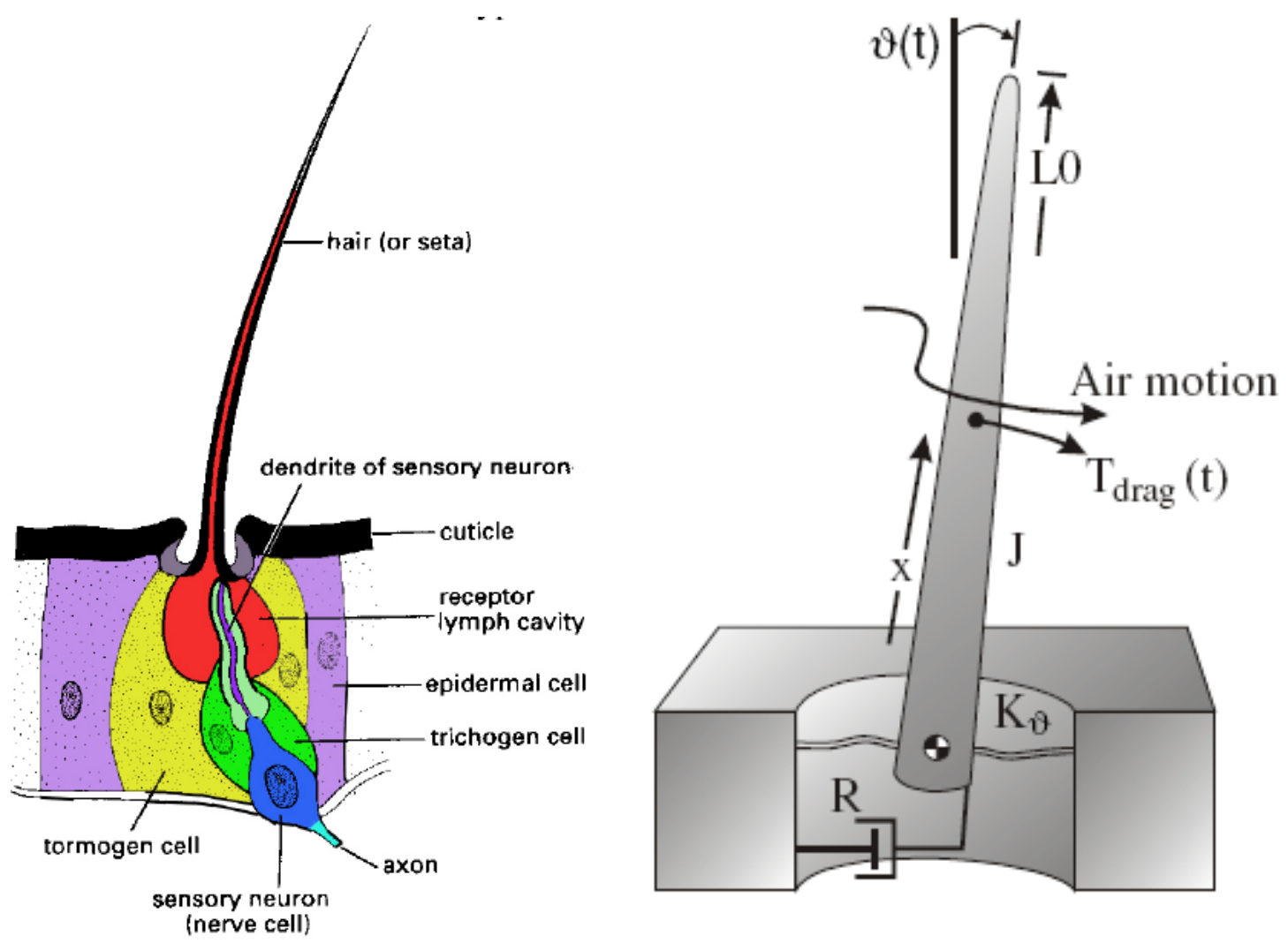

Figure 9 Left: An illustration of insect hair sensor (adapted from [5]); Right: its mechanical model (adapted from [6]), reproduced with kind permission from the authors of [6] and IOP Publishing Ltd., UK

A biomimetic silicon-based sensor, based on this hair structure has been developed at Twente University, The Netherlands. The basic structure can be adapted to silicon using a 
thin-film membrane and an SU-8 hair. A cross section of such a device is given in Figure 10, with a SEM photograph of fabricated devices in Figure 11.

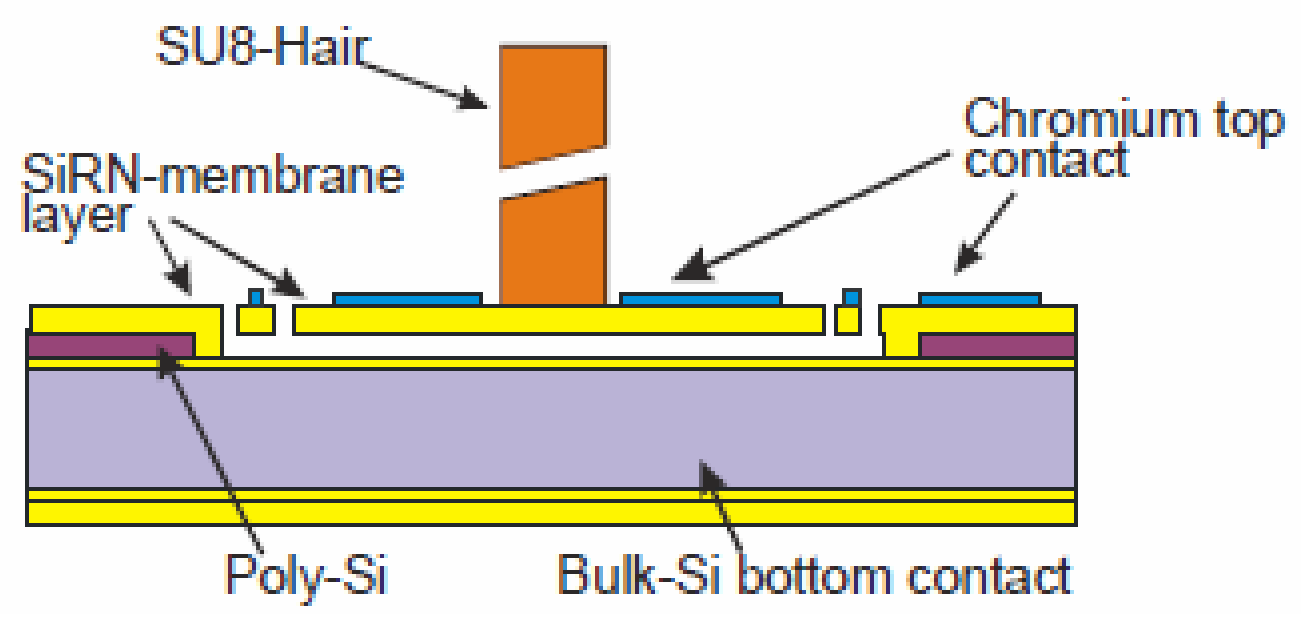

Figure 10 Schematic representation of artificial biomimetic flowsensors based on cricket mechanosensory hairs [7].

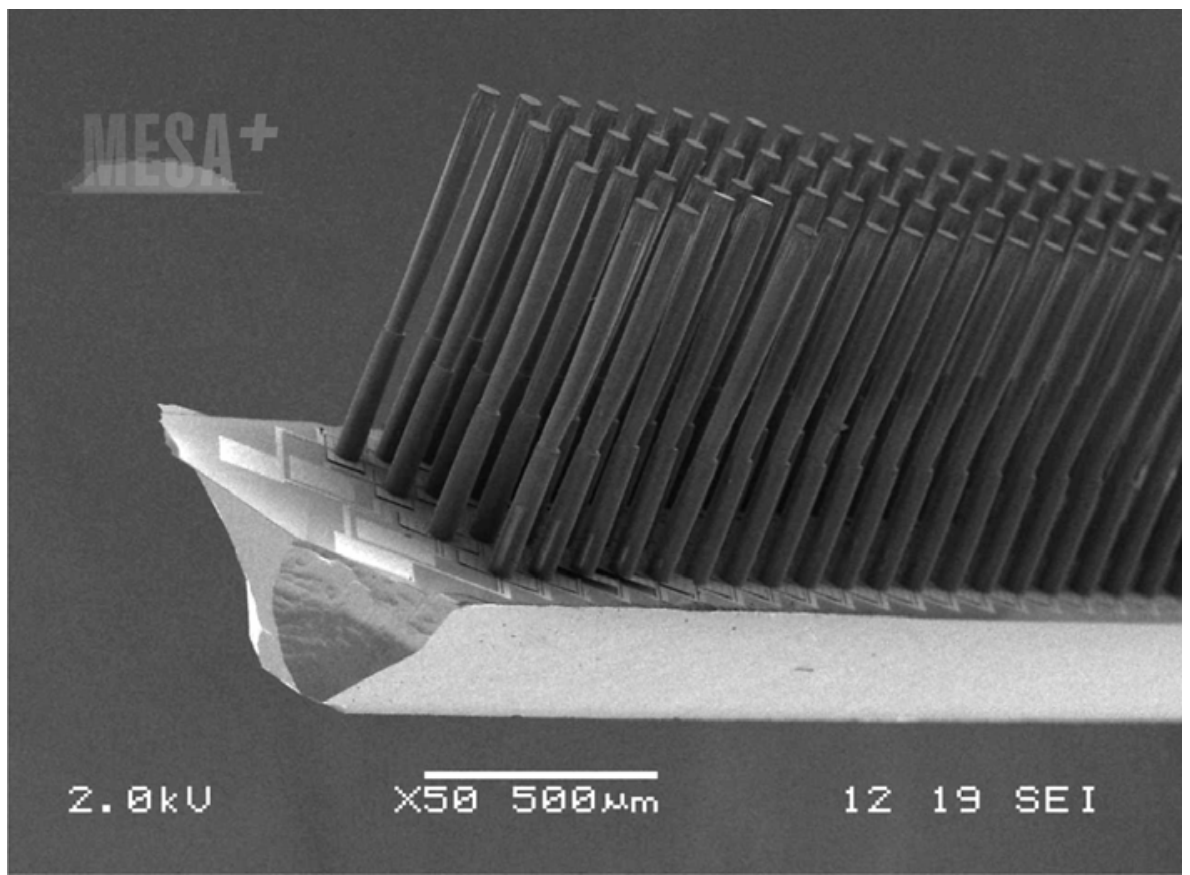

Figure 11 SEM photograph of a fabricated array [7]. Reproduced with kind permission, Gijs Krijnen.

A second generation of this sensor is given in Figure 12 and Figure 13 [8]. One of the features of this new device is the two-step SU-8 pillar. 


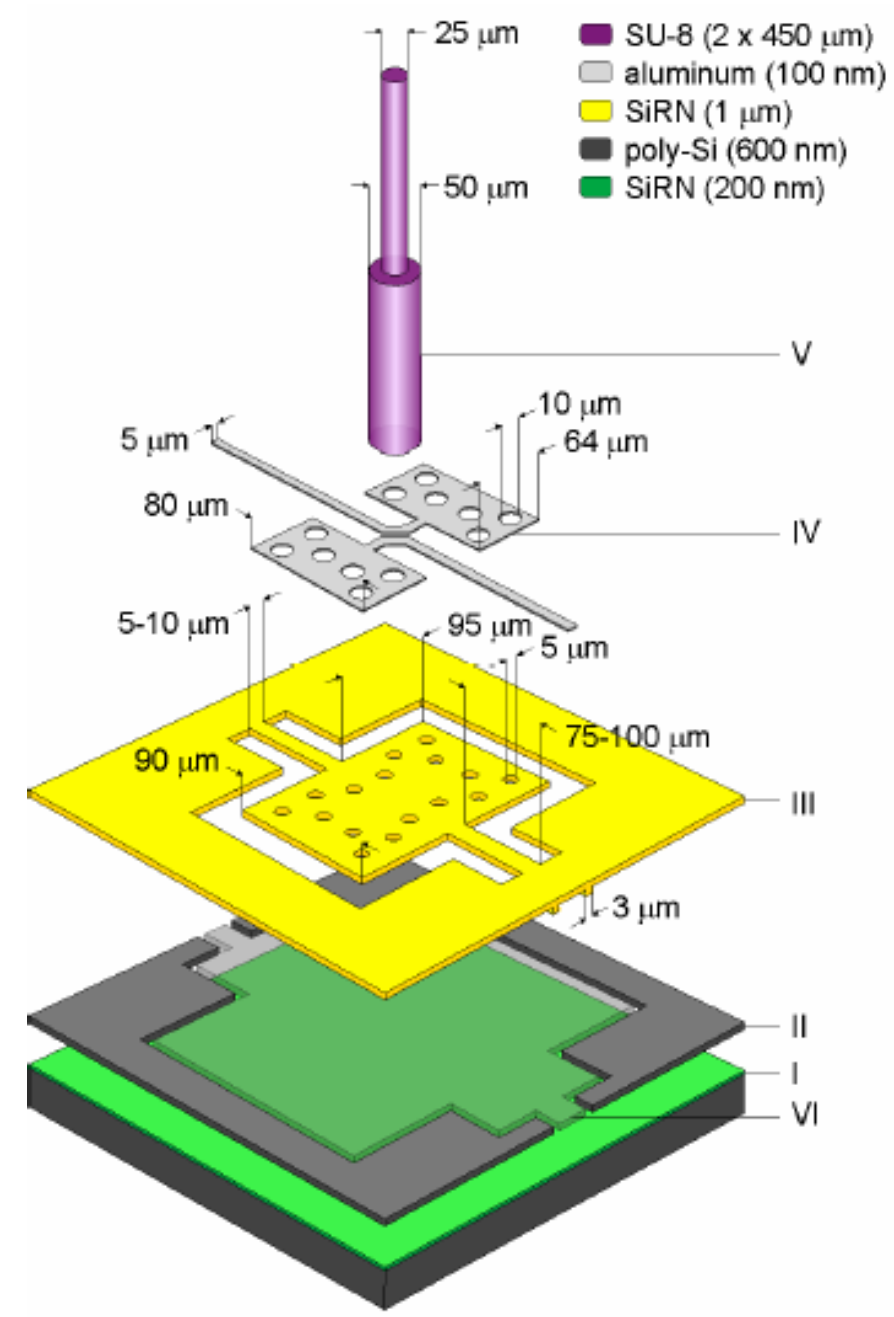

Figure 12 Schematic representation of the fabrication of the artificial hair sensor. (I) LPCVD SiN; (II) LPCVD sacrificial poly-Si layer; (III) LPCVD SiN layer defining the membrane/torsion beam structures; (IV) aluminium top electrodes; (V) two-layer SU-8 hair [8.] Reproduced with kind permission, Gijs Krijnen

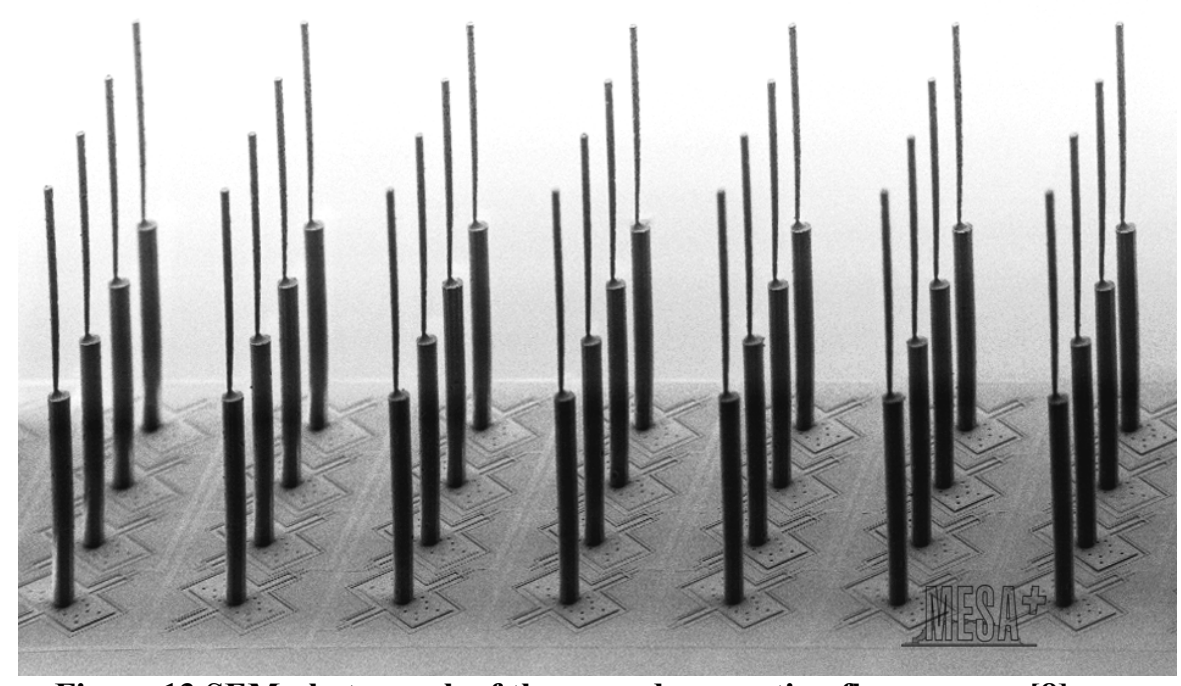

Figure 13 SEM photograph of the second generation flow sensors [8]. 


\section{Gyroscope}

The gyroscope is an important sensor for many animals. The human ear contains a gyroscope for balance, which contains three perpendicular rings filled with fluid. The common housefly also has a gyroscope for balance. The gyroscope is based on the inverted pendulum and has two resonant modes, which correspond to the two beat frequencies of the fly's wings. The structure of the device can be seen in Figure 14.
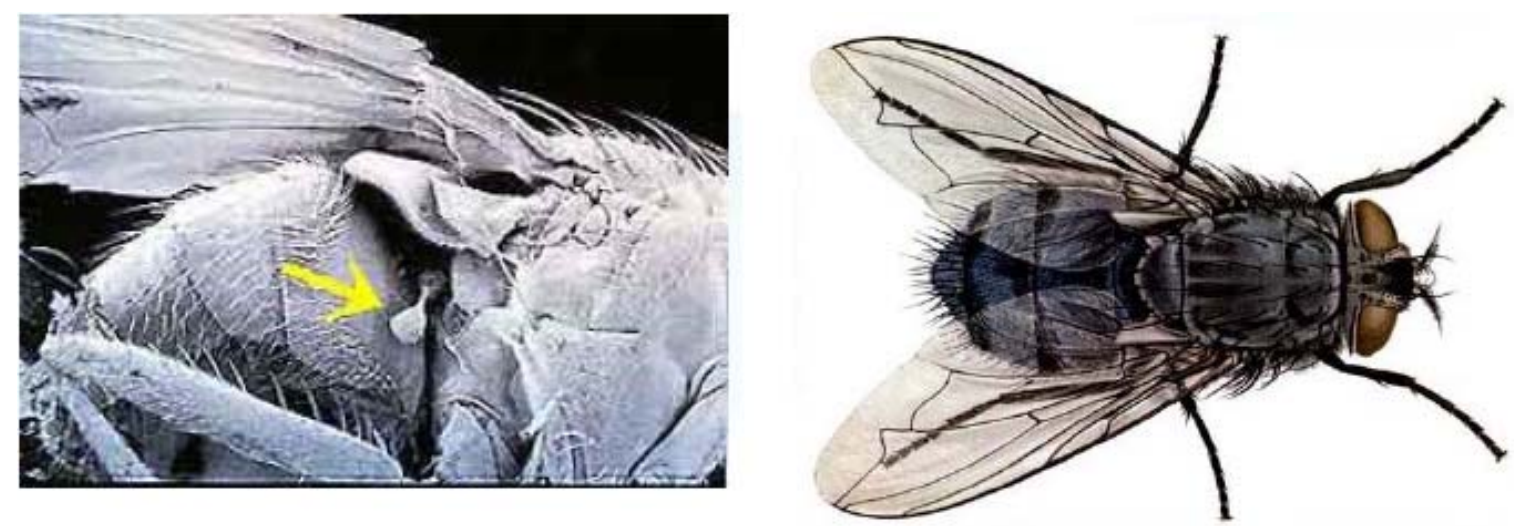

Figure 14 Gyroscope found under the wing of a fly.

The exact structure of the device is difficult to copy directly in silicon, but features can be used, in particular the inverted hammer aspect. This is illustrated in Figure 15 [9]. This has some similarities to the flow sensor shown above. However, in this case a larger head is used to increase sensitivity. This device uses a PECVD SiC membrane and springs. This is a low-temperature process, and thus aluminium could be used as the bottom electrode. The cross-section of the device is given in Figure 16.

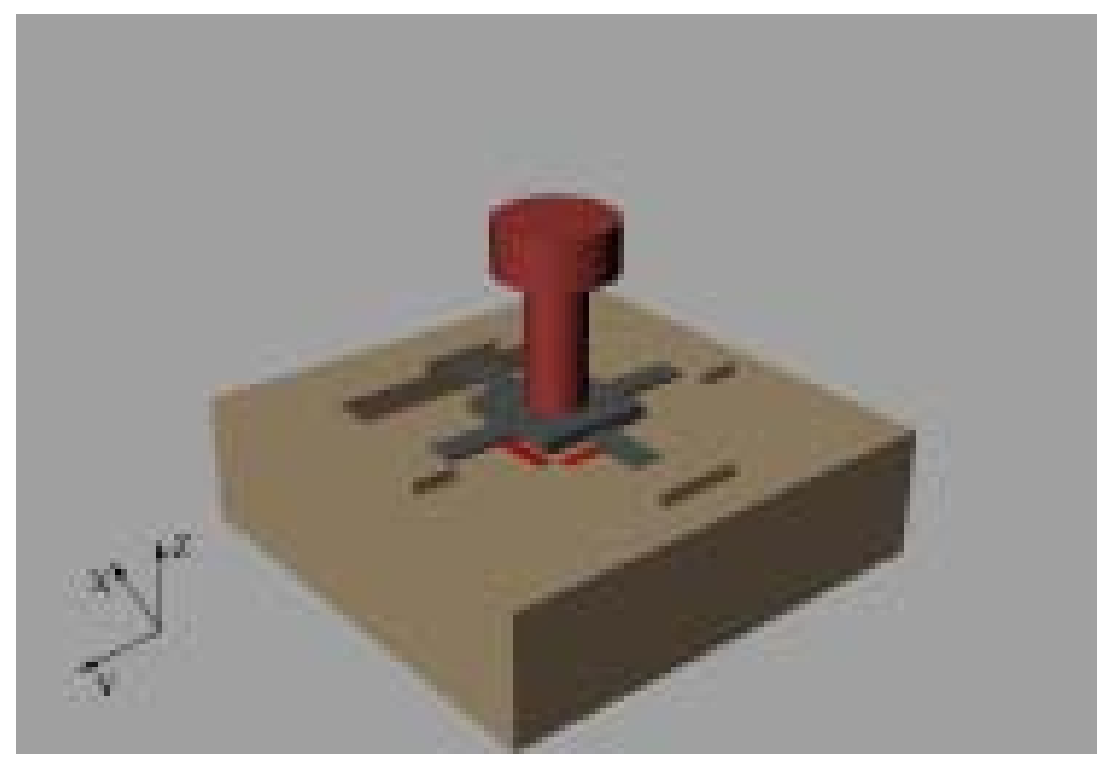

Figure 15 Surface-micromachined bio-inspired gyroscope with a standing pillar and suspending beam 


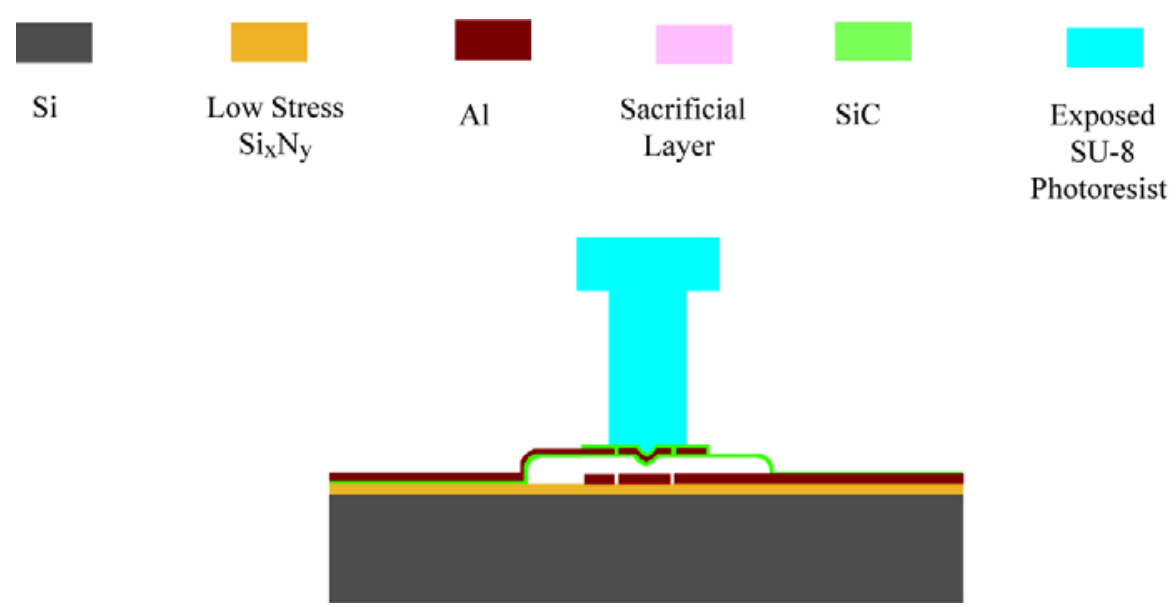

Figure 16 Cross-section of the gyroscope, showing the layers used [10].

\section{$\underline{\text { Infrared sensor }}$}

Nature has found its own way for sensing and imaging infrared thermal radiation. Some examples can be mentioned here. Boid snakes possess infrared (IR) imaging receptors called pit organs, which work along with their visual and other sensory systems to enable them to detect, locate and apprehend prey. Vampire bats possess IR pit organs, which enable them to locate blood-rich locations on their prey by detecting the IR radiation they emit. Forest fire-seeking beetles (Melanophila acuminata), which lay their eggs in trees freshly killed by forest fires, detect forest fires with IR detecting pit organs. The ability of the beetle to detect IR radiation has been developed to detect forest fires. The forest fires emit IR radiation that the beetle detects via a specialized IR sensor known as the "IR pit organ". Each beetle possesses two pit organs on its body which are filled with around 60 nodules (Figure 17). Each of these is a thin-shelled cavity that is filled by a sphere. The bottom of the sphere is attached to sensory neurons. When struck by radiation of around $3 \mu \mathrm{m}$ wavelength (strongly emitted by a forest fire) the nodule's shell is heated and slightly deformed by expansion, shifting the sphere and exciting the neurons, and making the insect aware of the heat source

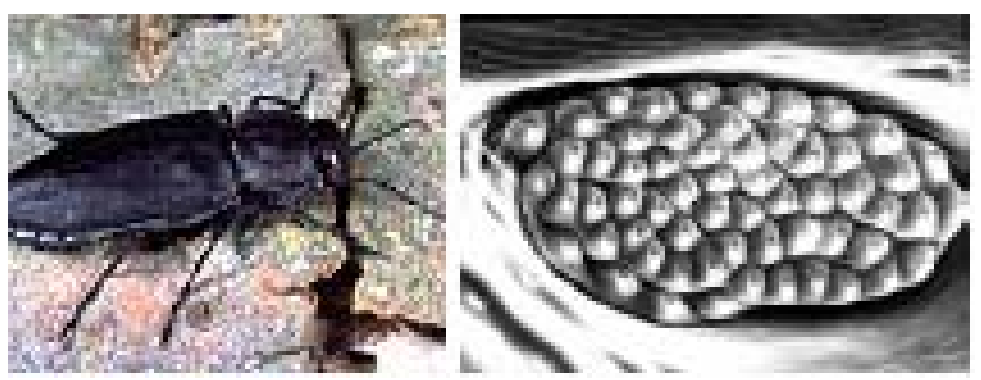

Figure 17 IR detection system of the beetle: the pit organ filled with sensilla.

This structure is perhaps difficult to reproduce in silicon, but the idea of measuring expansion to absorption of infrared can be used. Silicon nitride, which is a commonly used material in silicon processing, is a good absorber of infrared in the range from 7$14 \mu \mathrm{m}$ 


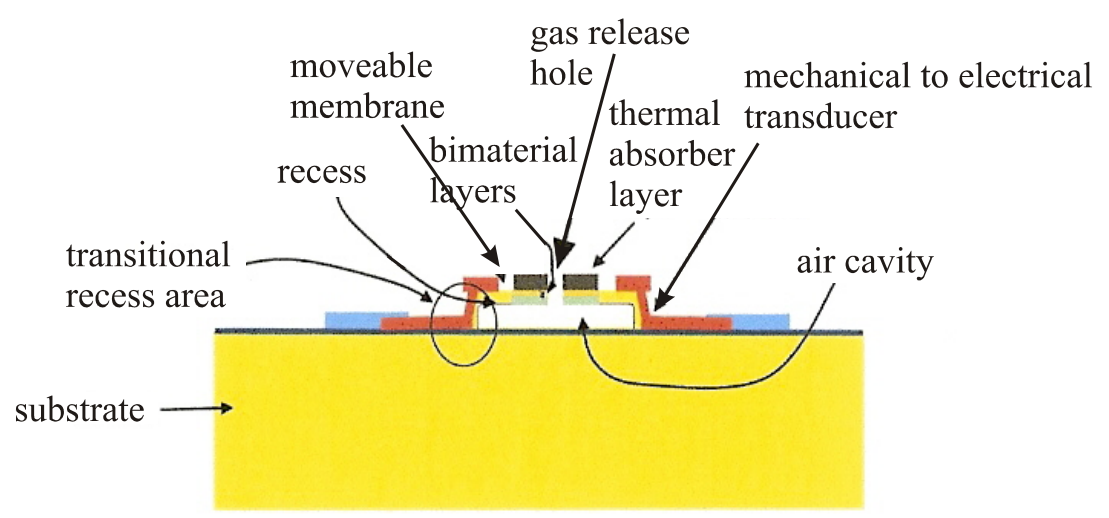

Figure 18 Cross-section of the silicon version of the infrared sensor.

\section{Conclusions}

This paper has given only a few examples of how we can learn from nature. In natural systems from insects to whales contain complete sensor and actuator systems. Each one is optimised for the application and the size, weight requirements of the creature. In some cases, sensors have been developed which use principles used in nature, where the researchers were unaware of the natural system. In other cases, new sensors have been developed through studying the natural systems and understanding the function of each part of the structure.

\section{Acknowledgments}

The authors would like to thank Prof Julian Vincent for the many discussions over the years, Prof Gijs Krijnen for a number of figures and collaboration of DIMES members who have helped over the years in fabricating some of the devices described in this paper.

\section{References}

1. S.N. Zill and E-A. Seyfarth, Sci.American., July 1996, pp. 70-74, 1996.

2. N. Matushkina, S. Gorb, J. of Insect Phys., vol. 48, pp. 212-219, 2002.

3. T.A. Keil, Microsc. Res. and Tech., 1997, 39: 506-531

4. U. Grünert and W. Gnatzy, Campaniform sensilla of Calliphora vicina (Insecta, Diptera) II. Typology, Zoomorphology, vol. 106. pp. 320-328, 1987.

5. D.L.A. Underwood, Biology 316 - General Entomology Lecture Note, Univ. of California, Davis, California

6. M. Dijkstra, J.J. Van Baar, R.J. Wiegerink, T.S.J. Lammerink, J.H. De Boer, and G.J.M. Krijnen, J. of Micromech. And Microeng., 2005, 15: S132-S138

7. Krijnen, G.J.M. and Lammerink, T.S.J. and Wiegerink, R.J. and Casas, J. Proceedings In: IEEE Sensors 2007, 28-31 Oct 2007, Atlanta, USA. pp. 539-546.

8. Bruinink, C.M. and Jaganatharaja, R.K. and de Boer, M.J. and Berenschot, J.W. and Kolster, M.L. and Lammerink, T.S.J. and Wiegerink, R.J. and Krijnen, G.J.M. Proceedings IEEE MEMS, 25-29 Jan 2009, Sorrento, Italy.

9. D.H.B. Wicaksono, Y. Chen and P.J. French, Proceedings ICEEI, Bandung Indonesia, June 2007, pp 226-229.

10. D.H.B. Wicaksono, $\mathrm{PhD}$ thesis, TU Delft, The Netherlands 